\title{
Una épica relevante: la cultura legal argentina y las escenas judiciales del Martín Fierro
}

Daniela Dorfman

Recebido em: 26 de janeiro de 2020

Aceito em: 20 de março de 2020
Daniela Dorfman es doctora en Literaturas Latinoamericanas por Boston University, becaria postdoctoral de CONICET y profesora de literaturas y culturas latinoamericanas en grado y posgrado en diversas universidades argentinas y del exterior. Desarrolla su investigación en el Instituto de Literatura Hispanoamericana de la UBA y se dedica a los estudios culturales y problemas de Derecho en y a través del arte y la literatura en América Latina y, específicamente, en el Cono Sur. Contato: dorman1@bu.edu Argentina 
PALABRAS CLAVE:

Literatura argentina; gauchos; ley; justicia; siglo XIX.

KEYWORDS: Argentine literature; gauchos; law; justice; 19th Century.
Resumen: Este artículo investiga los modos en que la literatura argentina del siglo XIX articula las relaciones de los habitantes con el Estado y con la ley. Interroga las maneras en que la literatura argentina representó e intervino en lo que Lawrence Friedman Ilama la "cultura legal": el conjunto de opiniones, expectativas, valoraciones, y actitudes de la sociedad hacia la ley. A partir del estudio de las escenas judiciales en el Martín Fierro, de José Hernández (1872, 1879), y la disputa que entabla con las nociones estatales de criminalidad y de justicia, mi argumento es que el texto fija una posición de la literatura respecto de la ley y de la justicia estatal que disloca el lugar simbólico de la criminalidad en la sociedad, y así fundan un lugar de enunciación específico de la literatura argentina que continúa vigente hasta hoy.

Abstract: This article looks at the ways 19th-century Argentine literature articulates the relationship between the country's inhabitants, the State and the law. It interrogates the ways in which Argentine literature represented and intervened in what Lawrence Friedman has called "legal culture": the ensemble of a society's opinions, expectations and attitudes regarding the law. Through a study of judicial scenes in Martín Fierro by José Hernández (1872, 1879) in which State notions of criminality and of justice are disputed, I argue that the text fixes a position of literature on the law and State justice that displaces the symbolic place of criminality in society, and establishes a site of enunciation specific to Argentine literature that continues to operate into the present. 


\section{Cultura legal y literatura Argentina}

En su ensayo "Nuestro pobre individualismo" (1946) Jorge Luis Borges se pregunta por qué, a diferencia de otras literaturas, en la argentina el héroe popular es el hombre solo que pelea con la partida policial: Martín Fierro, Juan Moreira, Hormiga Negra. Sostiene, entonces, que los argentinos no se identifican con el Estado, que el postulado hegeliano de que "el Estado es la realidad de la idea moral" les parece una broma siniestra y que, además, esto constituye una especificidad que distingue a los argentinos de los norteamericanos y de los europeos. Cuando las películas de Hollywood, dice, proponen a la admiración el caso de un hombre que busca la amistad de un criminal para entregarlo después a la policía, el argentino "para quien la amistad es una pasión y la policía una maffia, siente que ese 'héroe' es un incomprensible canalla" (II, 34). ${ }^{1}$

En Law, Lawyers, and Popular Culture Lawrence Friedman (1989) presenta una teoría social que niega autonomía al Derecho: postula que la forma en que la cultura popular ${ }^{2}$ caracteriza a la ley interviene en la formación de cultura legal, y que la cultura legal así formada, lo que la gente piensa de la ley, determina el grado de desviación de la norma, es decir que constituye una fuente de la

1 En este ensayo -escrito durante la primera presidencia de Juan Domingo Perón- Borges está postulando un "individualismo argentino" para asignarle un uso político: resistir la intromisión del Estado en la vida de los individuos. En mi libro (en preparación) sobre escenas judiciales y relaciones con la ley en la literatura argentina del siglo XIX al XXI dedico un capítulo a las ideas borgesianas sobre la ley y su aspiración a una justicia desligada del Estado.

2 "Cultura popular" refiere en Friedman alternativamente a las normas y valores de la "gente común" (que define como "no intelectuales") y a la producción cultural no dirigida a la intelligentsia sino al público general. En este caso lo está usando en el segundo sentido (1989, 1579). 
efectividad de la ley. ${ }^{3}$ Por eso, en tanto -como sostiene Paul Kahn (1999)- el poder de la ley no se mide por las acciones judiciales sino que es producto de la imaginación, el estudio de la cultura legal de una sociedad resulta crucial para entender su comportamiento y el funcionamiento de su sistema judicial. En este sentido, las producciones culturales, aun sin ser idénticas a la opinión pública -ya que hay tabúes y factores estéticos, culturales, políticos, y comerciales operando como selectores del contenido- constituyen una vía de acceso, un testimonio del valor y el lugar que una sociedad determinada otorga a la ley.

Este artículo estudia los modos en que el texto fundacional de la literatura argentina articula, en el siglo XIX, la relación de los habitantes con el Estado y con la ley $y^{4}$ las maneras en que, en este período de constitución simultánea de instituciones y discursividades, la literatura representó e intervino en la conformación de lo que -siguiendo a Friedman- llamo "cultura legal", es

3 No solo, como afirma Friedman, "the public learns its law from the evening news" (1605), también el sistema legal recurre a las representaciones de la cultura popular para darse forma a sí mismo: en marzo de 2015, cuando se inicia en la Provincia de Buenos Aires un nuevo sistema de juicio por jurados, una nota de Diario Clarín firmada por Fernando Soriano explica el nuevo sistema remitiendo a productos de la cultura popular: "En películas y series extranjeras hay muchos ejemplos de cómo funciona este sistema", incluso sostiene que "el entrenamiento que se les está dando a fiscales, abogados y jueces se hace a partir de estas ficciones. Por ejemplo, el film 'El Sospechoso' (1987), de Peter Yates, en el que la actriz Cher acepta y recusa candidatos a partir de las respuestas a sus preguntas." (Clarín, viernes 13 de Marzo de 2015).

4 Aunque las opiniones sobre la ley, su legitimidad, y su autoridad, y la actitud hacia ella, varían según cómo se segmenten los grupos sociales por edad, género, clase social, educación y otras variables, es posible distinguir patrones de comportamiento y valoraciones predominantes y sistemáticos en grupos heterogéneos. Lawrence Friedman (1969) contrasta la buena disposición a pagar impuestos en Estados Unidos con la reticencia que produce en Italia, y Susan Silbey (2010) llama la atención sobre la repetida referencia en estudios recientes a "Cultures of Legality", culturas en que la invocación de la ley acarrea legitimidad. 
decir, el conjunto de opiniones, expectativas, valoraciones y actitudes de la sociedad hacia su sistema legal y judicial.

Para pensar eso voy a centrarme en las escenas judiciales representadas en el Martín Fierro, de José Hernández $(1872,1879)$. Dadas las características del sistema legal y judicial en la época, su carácter embrionario, no estabilizado sino todavía en formación, tomo como escenas judiciales enfrentamientos en que una de las partes representa la ley o actúa en su nombre, aun si no constituyen un juicio como lo concebimos hoy, porque dentro del sistema que las sostiene se postulan como situaciones judiciales, si no legítimas, al menos válidas, cuyo estatuto legal es una de las problemáticas que voy a interrogar. Mi argumento es que estas escenas crean un espacio de significación alternativo en el que se entabla una batalla simbólica con el sistema de justicia estatal y que, en este sentido, son definitorias porque fijan una posición de la literatura respecto de la ley y del aparato judicial del Estado, y fundan un lugar de enunciación sustentado en una cultura legal específica de la literatura nacional y del imaginario argentino que continúa vigente hasta hoy. ${ }^{5}$ En los años que comprende este artículo, aunque ya se habían sancionado la Constitución Nacional (1853), el código rural (1865) y el civil (1871),

5 En Nightmares of the Lettered City Juan Pablo Dabove postula que en la literatura argentina los héroes nacen en el enfrentamiento con la policía y, en ese sentido, considera que la Ida de Martín Fierro abre el camino a Roberto Arlt, a los Montoneros de los años '70 y a Plata quemada (1997) de Ricardo Piglia. En esa genealogía yo agregaría Juan Moreira de Eduardo Gutierrez (que Dabove excluye deliberadamente) ya que impuso la historia del gaucho que enfrenta a la partida no solo en la literatura, sino en la conducta de los paisanos, que eran detenidos por lo que en los prontuarios policiales figura como el delito de "hacerse el Moreira": vestirse como Moreira y desafiar a la policía (Balderston 2000, 44). 
éstos se aplicaban en combinación con normas y disposiciones provenientes de códigos anteriores (del Derecho español y la Recopilación de las Leyes de Indias), es decir que coexistían normas contrapuestas y los jueces de paz seleccionaban de entre la multitud de códigos vigentes qué leyes aplicar y, sobre todo, a quién aplicarlas. En este contexto el Martín Fierro realiza demandas de inclusión, de reconocimiento jurídico; visibiliza así aquello que el sistema jurídico vigente excluía, sus "residuos" (el término es de Wai Chee Dimock), construyendo un contra-discurso que se afirma en las capacidades jurisgenéticas de la literatura, en su potencialidad de crear significados legales. ${ }^{6}$

De esta manera, durante el "salto modernizador" (Ludmer, 1994, 102) en que tiene lugar el proceso de instauración de la ley y de formación del sistema jurídico y del aparato judicial, la literatura argentina también en formación no solo problematiza la relación de los argentinos con el Estado, específicamente con su sistema legal, sino que textos como el Martín Fierro (también El matadero, de Echeverría y Juan Moreira de Eduardo Gutiérrez) conjuran la "demonización de la escritura" (Alonso, 1994, 286) con que Ángel Rama -al incluir todo el mundo escrito en la ciudad letrada, en inescapable complicidad con el poder- despoja a los escritores de la posibilidad de una práctica contestataria u opositora. Estos textos inaugurales y canónicos de la literatura nacional asumen un lugar de enunciación que desestabiliza el vínculo entre escritura y poder y, visibilizando los mecanismos con que el Estado criminaliza, marginaliza y excluye a sus otros, disputan las nociones

6 "Jurisgenesis" es la creación de significado legal por parte de personas o grupos no autorizados, e incluye la obediencia a leyes no-oficiales (Haritatos, P.) 
de criminalidad y de justicia que el Estado intentaba imponer, dislocando el lugar simbólico de la criminalidad en la sociedad.

\section{El Estado - llegalizar para ApLICAR LA ley}

El esquema interpretativo tradicional leía el período posterior a la colonia como un vacío constitucional. Revisiones posteriores, sin embargo, echaron luz sobre la existencia de una "constitución material", un conjunto de normas y disposiciones provenientes de distintos códigos que, aunque no estuvieran recogidos en un texto único escrito, regían las relaciones sociales y la actividad política. Esto favorecía una aplicación selectiva de la ley debido a la dificultad para precisar el contenido de una constitución no escrita que, además, abarcaba normas que iban desde las partidas de Alfonso X del siglo XIII, hasta la Recopilación de las Leyes de Indias del siglo XVII, y permitía la coexistencia de normas contrapuestas.

Pero aun después de la sanción de la Constitución Nacional (1853), el código rural (1865), el civil (1871) y el penal (1877) 7 , los juristas no abandonaron completamente la utilización del antiguo Derecho español y los jueces siguieron fundamentando sus decisiones con normas de los siglos XIII a XVII hasta ¡1931! (Chiaramonte n.p). En 1871, cuando se publica la Ida del Martin Fierro, aunque ya se ha implementado el Estado liberal, la administración de justicia está en manos de jueces de paz que seleccionan de entre una multitud de códigos vigentes qué leyes aplicar y deciden también

71886 para el territorio nacional. 
sobre quién aplicarlas. Es así que el sistema de justicia diferencial (para la ciudad y el campo) y selectivo (en su aplicación a ciudadanos, gauchos y extranjeros) constituye uno de los contextos específicos del género gauchesco.

La diferenciación jurídica entre las personas fue señalada por la poesía popular desde poco después de la Revolución de Independencia en 1810 (i.e Hidalgo), y después de la Batalla de Caseros (1852), que marca la caída de Juan Manuel de Rosas, empiezan a escucharse voces también en el Congreso, pero es con El gaucho Martín Fierro (1872) que adquiere visibilidad el mecanismo con que el Estado clasifica al gaucho como vago para ilegalizarlo y poder, entonces, aplicarle la ley. ${ }^{8}$ Giorgio Agamben (2007) llama "bando" a la estructura original por la cual la ley se aplica incluso a aquello que ella misma excluye de sí, que envía al bando, convierte en bandido, abandona. ${ }^{9}$ El Martín Fierro narra cómo el Estado envía al gaucho fuera de la ley y en

8 En septiembre de 1873, el Jefe de Policía de Buenos Aires pide más potestades para castigar a "vagos y malentretenidos" -eran "malentretenidos" los pobres del campo que participaran en fiestas y entretenimientos fuera de su partido de residencia (Sarlo y Gramuglio 43)-. Pero el fiscal J. S. Fernández responde que "La vagancia en sí misma, no es un delito y si se castiga es como medida preventiva” (Molas 590) haciendo manifiesta la lógica paranoica con que las leyes castigan según las fantasías de la clase dirigente.

9 Agamben dice que lo llama así desarrollando una sugerencia de J.-L. Nancy y que la palabra proviene de un antiguo término germánico que indica tanto la exclusión de la comunidad como la insignia del soberano y expresa el carácter soberano de la ley, el poder de incluir excluyendo (164). No encontré el término germánico al que se refiere Agamben, pero sí "bannan", que significa "hablar en público", y que los diccionarios etimológicos dan como origen de "proclama" y de "bandido", por lo que podemos pensar que estaría relacionado.

En un sentido similar, "bando" se llamó en el XIX a las proclamas con que las autoridades argentinas ordenaban a quien había caído fuera de su vista reaparecer ante ellas (Dabove, 2007, 8). Para una discusión del término y su relación con "bandido" ver Juan Pablo Dabove, Nightmares of the Lettered City. 
el mismo acto ocluye la existencia misma de un "afuera" de la ley porque incluso ahí, en su propio exterior, está la ley regulando. Catalogado de vago y enviado a pelear en la frontera, el gaucho es el sujeto al que -como dice Fermín Rodríguez- el Estado excluye por medio de la inclusión en el servicio de armas (305).

Ante el aspecto representacional de la ley, que es instituyente y que impone definiciones e imagina relaciones sociales, el Martín Fierro opone un gaucho representado como víctima más que como perpetrador (Caimari, 2004, 197), y de esa manera pone en evidencia el desajuste entre el sujeto histórico y su representación jurídica. En tanto las normas adquieren su significado de la épica que se elige hacer relevante para ellas (Cover, 1983, 4) y dado que las teorías de la causa son en su centro teorías sociales (Dimock, 1996, 161), este planteo según el cual el gaucho cae en la criminalidad llevado por las leyes oficiales del Estado disloca el lugar simbólico de la criminalidad en la sociedad y desestabiliza las nociones estatales de criminalidad y de justicia.

\section{La ida de Martín Fierro - El Derecho a la llegaldida.}

En la tradición del pensamiento político occidental, desde Aristóteles a Hegel, es en el Estado que el hombre se hace moral: mediante la internalización del Derecho la moral es impuesta por el Estado como el imperio de la ley. Pero a su vez, la ley positiva, escrita, del Estado, las leyes particulares que una comunidad se da a sí misma, entran en tensión con una ley no escrita, un Derecho natural que es anterior, superior e independiente del Derecho 
positivo, unas hipotéticas leyes basadas en un orden natural en relación a las cuales se mediría la justicia o injusticia de las primeras. Esta tensión es lo que dramatiza la lucha de Antígona, que considera "naturalmente justo" enterrar a su hermano, aunque vaya en contra de las leyes del Estado.

Si pensamos la legalidad que instituye el Martín Fierro desde este sentido naturalista de la ley, fundada en la idea de que -en palabras de San Agustín- "lex injustia non est lex" sino una perversión del Derecho y no habría, por lo tanto, obligación moral ni legal de obedecerla, podemos ver a Martín Fierro como alguien que mantiene su derecho de involucrarse en prácticas que chocan con la legalidad, y que lo que lo hace un justiciero no es la justicia o injusticia de sus actos sino su posición ante la ley. ${ }^{10}$

En las "culturas de la legalidad", donde se pondera la ley, el orden fundado por Martín Fierro es inconcebible porque lo que otorga a una regla

10 En su artículo "El desertor de fierro", Martín Kohan (2013) cuestiona que cuando Fierro provoca y finalmente mata no a "un justicia", responsable de sus injustos sufrimientos, sino a un pobre negro, cuando se vuelve a su vez victimario de ese otro, la injusticia padecida no lo convierte en justiciero sino en un reproductor de injusticias; Beatriz Sarlo (2003) se pregunta por la moral en un mundo como el de Fierro donde, en ausencia de las instituciones de la justicia, el coraje es lo que permite responder a la deshonra y la provocación, y piensa las condiciones, las circunstancias de excepción, que hacen que suspendamos nuestro juicio moral (192); y Borges y Bioy Casares (1955) señalan que aunque la lógica y el texto permitirían definir a Martín Fierro como pendenciero, borracho y asesino, estas definiciones no se corresponden con el concepto que tenemos de él, y que "no hay argentino que no las sienta como una especie de blasfemia o de irreverencia” (Poesía Gauchesca XXI); por último, Martínez Estrada (1958) lo explica diciendo que poseemos de Martín Fierro dos imágenes: la que formamos mentalmente por lo que nos confiesa de sí y de sus sentimientos y la que presenciamos en los actos que él mismo narra (I, 52). Mi argumento es que es precisamente el hecho de que la injusticia padecida lo convierte en un reproductor de injusticias, y de que cae en la criminalidad llevado por las leyes oficiales del Estado, lo que convierte incluso sus injusticias en actos de compensación (que es, en definitiva, el concepto de "reparación” y de "justicia” con que operan los sistemas judiciales). 
estatuto de ley y obligación de cumplirla es su fuente, el hecho de provenir de ciertas personas y de acuerdo con ciertos procedimientos, sin importar cuánto de ideales morales o de justicia le falte. A diferencia de estas culturas, donde la sola invocación de la ley ("It's the law") da legitimidad y clausura las discusiones, la Ida del Martín Fierro desafía no solo la letra de la ley sino la posición de enunciación que la sustenta, al Estado mismo como legislador, como dador de la ley, y articula una cultura legal de recelo hacia una justicia que utiliza la ley para perseguir y criminalizar. Por eso la literatura gauchesca representa una sociedad que, como dice Carlos Gamerro, es una Arcadia pastoril hasta que aparecen el juez y la policía (47).

El Martín Fierro transcurre en un contexto histórico-social en que la expansión de la vigilancia estatal sobre la población rural multiplicaba las instancias en que los paisanos confrontaban autoridades legales y policiales cara a cara y los ponía en creciente contacto con una ley punitiva que criminaliza, persigue y recluta. ${ }^{11}$ Estudiosos del crimen y de la justicia en Europa desarrollaron lo que llaman una "Reluctant litigator theory" y explican que en la Europa de entre la Edad Media y finales del siglo XIX, en la transición de una justicia restitutiva realizada por las comunidades locales a una justicia punitiva monopolizada por los Estados, los miembros de la sociedad civil se hicieron reticentes a involucrarse en asuntos judiciales porque el aumento en la intervención del Estado significaba también la criminalización de ciertos grupos (Barreneche, 1997, 6). Osvaldo Barreneche

11 Martín Fierro se queja: "Estaba el gaucho en su pago/con toda siguridá;/pero aura... ¡barbaridá!,/ la cosa anda tan fruncida,/ que gasta el pobre la vida/ en juir de la autoridá.” (II-26) 
postula que esto es lo que pasó también en el Río de la Plata donde, dado que gente involucrada inicialmente como testigo era a veces penada por los oficiales, las autoridades tenían problemas para obtener cooperación de los ciudadanos (1997, 45-46, 264).

Desde 1824, cuando se establece que la única prueba admitida en favor de quienes fueran aprehendidos como vagos es la palabra del juez de paz, el gaucho queda a merced de la simpatía u odio de las autoridades locales ("A mí el Juez me tomó entre ojos/ en la última votación:/ me le había hecho el remolón/ y no me arrimé ese día,/ y él dijo que yo servía/ a los de la esposición" (dice Martin Fierro, III-27). En 1827, cuando la ley autoriza al gobierno a reclutar "por los medios que considere más convenientes" cuatro mil hombres, los paisanos huyen lo más lejos posible del juez y del comisario, y ya para 1890 la policía controla los sistemas de vida y desplazamientos de los hombres sin recursos y, según el propio ministro de gobierno, era "casi un enemigo del ciudadano y el ciudadano odiaba a la policía y siempre estaba en contra de ella" (Rodríguez Molas, 1968, 151 y 458).

Martín Fierro presenta a la partida policial como enemiga del héroe popular, del gaucho perseguido cuyas penas son resultado del aparato represivo ("Antes de cair al servicio/tenia familia y hacienda;/ cuando volví, ni la prenda/ me la habían dejao ya. / Dios sabe en lo que vendrála parar esta contienda" IX-40) y el enfrentamiento de héroe y partida policial produce un quiebre en la identidad entre ley y justicia. Acá es donde Martín Fierro se hace justiciero, en la lucha contra la partida policial, en el enfrentamiento con la ley injusta, que hace que la justicia quede identificada con la voz, historia 
y representaciones del gaucho (Ludmer, 2012, 229). Esto es simbolizado en el cruce del Sargento Cruz cuando, como analiza Josefina Ludmer, en el momento crucial, el momento del enfrentamiento entre el bandido y la policía, los dos gauchos se encuentran, Cruz de un lado y Fierro del otro, y hacen alianza. En lo que es, sin duda, la escena judicial más recordada y celebrada de la literatura argentina hasta el día de hoy, el Sargento Cruz al grito de "Cruz no consiente/que se cometa el delito/ de matar ansí a un valiente!” (IX-40), invierte la categoría de delito y se cruza de bando para pelear con Martín Fierro en contra de la partida policial que él mismo comandaba $(2012,233)$.

Identificados con la justicia, Cruz y Fierro deciden entonces irse con los indios, donde "no alcanza la facultá del Gobierno", en busca de la seguridad que ahora no tienen y, desde entonces, la pelea con la policía será la escena en que los héroes nacen en la lucha contra la opresión y la injusticia y tendrá, como establece Juan Pablo Dabove, un lugar particular en la cultura argentina $(2007,180)$.

\section{La Vuelta de Martín Fierro - El auto-enjuliciamiento}

El sistema de reclutamiento no cambia hasta 1901 con la ley de servicio militar obligatorio, pero Martín Fierro vuelve en 1879 y, aunque la tesis central de la $I d a$ era su inocencia, su no culpabilidad, Martín Fierro viene ahora a purgar sus delitos. Los siete ańos transcurridos, las once ediciones de El gaucho Martín Fierro, las presiones políticas y las críticas que provocó, 
proporcionaron a José Hernández un sentido jurídico del poema y en 1879, desde la legislatura de la que ahora es parte, escribe lo que algunos ven como una versión taimada, atenuada, de la misma crítica social de la Ida y otros como la conformación e incorporación del gaucho al pacto civilizatorio, pero todos lo reconocen como libro didáctico, como la introducción de la voz del Estado en la literatura gauchesca.

Martín Fierro vuelve del desierto vencido, no a actuar sino a narrar sus desventuras e inculcar la ley. Cuando al llegar descubre que, muerto el juez, nadie recuerda ya los hechos, él mismo realiza un auto-enjuiciamiento que pone bajo la lupa los enfrentamientos de la Ida: recuerda la muerte del moreno y reconoce su imprudencia ("Aunque si yo lo maté/mucha culpa tuvo el negro./Estuve un poco imprudente,/puede ser, yo lo confieso"), excusa la pelea con el protegido del comandante en la pulpería ("Él, engreído, me buscó,/ yo ninguna culpa tengo;/ él mesmo vino a peliarme,/ y tal vez me hubiera muerto") y, finalmente, explica el enfrentamiento con la partida ("Esa vez me defendí/ como estaba en mi derecho,/ porque fueron a prenderme/ de noche y en campo abierto/ (...) de un modo que daba miedo.") (XI-73). En algunos momentos utiliza el código de la ley oral para justificarse ("Pero él me precipitó,/ porque me cortó primero,/ y a más me cortó la cara,/ que es un asunto muy serio.") pero su lengua y su voz coinciden ahora con la ley escrita del Estado. Martín Fierro se mira y se juzga a sí mismo desde, no en contra de, las nociones estatales.

El auto-enjuiciamiento que constituye la escena judicial de la Vuelta no es ya la escena en que los héroes enfrentan la ley, pelean contra la opresión o la 
injusticia. Sin autoridades ni representantes del Estado, este juicio revierte la escena judicial de la Ida: Martín Fierro acepta ahora la restricción de la ley para acceder a su protección y ya no distingue entre leyes justas e injustas, la ley es la ley estatal y hay que obedecerla. Esta reconciliación, esta identificación de Martín Fierro con la ley del Estado, la asunción de su voz para justificar crímenes que "le habíamos perdonado porque le eran extraños" hiere el sentido de justicia que había instaurado la $I d a$, por eso Martínez Estrada se queja de que "a su regreso se engañe y nos engañe excogitando penosa y dolorosamente atenuantes a sus actos" (I, 60, subrayado mío), porque asumir la voz de la ley estatal, su lógica y su noción de justicia, significa aceptar una culpabilidad que modifica la definición de criminalidad que el texto mismo había instaurado. ${ }^{12}$ Martín Fierro reconoce los hechos que según las nociones estatales lo hacen culpable, da atenuantes, y no se declara culpable ni inocente porque lo central, lo que es políticamente relevante en la Vuelta, es la reconversión del gaucho, su aceptación de la moral del Estado para construirse como sujeto obediente e incorporarse a la comunidad.

La Ida, al afirmar que el Estado cambiaba las definiciones de legalidad para poder ilegalizar al gaucho, exaltaba la legitimidad de la violencia popular y el Derecho promovido por ésta; la Vuelta, en cambio, se acopla a las definiciones estatales y suspende el derecho de Fierro a la violencia. A pesar de sus diferencias, sin embargo, las dos partes terminan en la exclusión del gaucho: en la $I d a$, Cruz y Fierro se van a vivir con los indios, donde no llega

12 De hecho, Martínez Estrada encuentra en el manuscrito numerosas enmiendas que sugieren la dificultad de Hernández para realizar esta operación. 
la ley (“...y hasta los indios no alcanza/ la facultá del Gobierno./ (...) Alcemos el poncho y vamos./ (...) Allá habrá seguridá,/ ya que aquí no la tenemos" XIII-46); en la Vuelta, después de los consejos con que Fierro inculca la ley ("El trabajar es la ley", "A ningún hombre amenacen", "El hombre no mate al hombre/ ni pelee por fantasía" XXXII- 99 y 100) él, sus dos hijos, y el hijo de Cruz, recientemente reencontrados, se dispersan ("Después, a los cuatro vientos/ los cuatro se dirigieron") y cambian de nombre ("convinieron entre todos/ en mudar allí de nombre").

Como muestra la conclusión del narrador -"Vive el águila en su nido,/ el tigre vive en la selva,/ el zorro en la cueva ajena,/ y en su destino inconstante/ sólo el gaucho vive errante/ donde la suerte lo lleva" (XXXIII-101)-, la integración de Martín Fierro en la Vuelta no es total y, en ese sentido, termina como la Ida: en una marcha a lo desconocido que reafirma la imposibilidad del gaucho de integrarse en el proyecto de país (Sarlo y Gramuglio, 1968, 42). El gaucho, arrojado fuera de la sociedad y producido como su exterior constitutivo, ya no podrá pasar a ser completamente interno, pero retornará para acechar las políticas predicadas sobre la base de su ausencia. Como observan Ernesto Laclau y Chantal Mouffe, ${ }^{13}$ el retorno de las exclusiones mediante las cuales se constituyeron las organizaciones democráticas, fuerza una expansión y una rearticulación de las relaciones sociales: la figura del gaucho operará, entonces, políticamente como encarnación de demandas de otros excluidos, demandas de inclusión y de expansión de la esfera de la

13 Citados por Judith Butler en "Replantear el universal" $(2011,19)$. 
legalidad del Estado hacia el sujeto popular. La estructura esencial del planteo de la literatura gauchesca va a retornar, así, en la cultura argentina en diversos soportes para presionar la apertura de nuevos horizontes conceptuales.

\section{CONCLUSIONES}

Como la propiedad específica del poder simbólico es que solo puede ser ejercido mediante la complicidad (inconsciente) de quienes son dominados por él, el Derecho solo puede ejercer su poder en la medida en que se desconoce su arbitrariedad. En este sentido Pierre Bourdieu (1987) destaca el alcance que los procedimientos tienen en la eficacia de la ley y los considera cruciales para obtener y sostener la aprobación social porque son tomados (sin importar cuán ilógicamente) como signo de imparcialidad y neutralidad, es decir que la eficacia de la ley y, por lo tanto, la estabilidad del campo jurídico dependen de esta "racionalidad formal". Por eso dice que debemos otorgar realidad social al poder simbólico que la ley debe al efecto específico de los procedimientos y la codificación de comportamientos. ${ }^{14}$ Este es un problema recurrente cuando se piensa la ley y la justicia en Argentina y, como vimos, el Martín Fierro estructura instancias judiciales ilegítimas, que no cumplen con los procedimientos, precisamente como una manera

14 Esto remite a la famosa fórmula con que Pascal trastoca "escandalosamente" el orden de las cosas cuando dice "Arrodilláos, moved los labios en oración, y creeréis", que Althusser recuerda en su postulación de una existencia material de la ideología en nuestros actos en tanto están insertos en prácticas reguladas por rituales que son dictados por la clase dominante (50). 
de señalar la justicia estatal como una farsa que simula la administración de una justicia que no realiza.

Martín Fierro acusa a la administración existente y los modos adjetivos de la justicia e instituye, entonces, una legalidad alternativa. Fundada en una concepción iusnaturalista de la justicia, en un hipotético "Derecho Natural” que sería superior e independiente del Derecho Positivo, la Ida afirma que lex injustia non est lex y, poniendo en evidencia aquello que la ley excluye, mide la legitimidad de la ley, del sistema jurídico-judicial que la implementa y del Estado que la sostiene de acuerdo a su resultado último, es decir, a un sentido naturalista que organiza nociones sustantivas de justicia. En la Vuelta Martín Fierro acepta la ley del Estado y su posicionamiento cambia: la justicia ya lo olvidó pero él se auto-enjuicia en otro proceso sin legitimidad oficial.

En toda simulación hay una disputa sobre la representación y un debate político, y la farsa de justicia que, según Hernández, monta el Estado hiere la noción misma de una "justicia estatal". La literatura muestra, entonces, la presencia de la ficción en lo real y en la política, y revela la construcción de la realidad desde el Estado y al Estado como conspirador que manipula y ordena las relaciones sociales (Piglia, n.d). Creo que la recurrencia de escenas judiciales en los textos fundacionales de nuestra literatura (como mencioné antes, además de en el Martin Fierro, también en El Matadero y en Juan Moreira la trama misma se elabora alrededor de la escena judicial) revela una preocupación por el modo en que se tramitan los conflictos y plantea preguntas sobre las ideas de justicia y el tipo de demandas que esto expresa 
y, también, sobre cómo y por qué la literatura procesa esas demandas y qué efectos extra-literarios puede producir.

En su organización de la justicia estatal como una farsa que abusa de los procedimientos y contradice la justicia sustantiva del Derecho Natural, el Martín Fierro manifiesta recelo y desconfianza hacia el sistema legal; y al oponerle una noción de justicia propia, funda un lugar de enunciación para la literatura nacional que, como sostuve anteriormente, va a contribuir a la conformación de una "cultura legal" específica de la literatura y el imaginario argentinos hasta la actualidad.

\section{REFERENCIAS BIBLIOGRÁFICAS}

Agamben, Giorgio. "El mesías y el soberano. El problema de la ley en Walter Benjamin”. In: La potencia del pensamiento. Buenos Aires: Adriana Hidalgo, 2007.

Alonso, Carlos. "Rama y sus retoños: Figuring the Nineteenth Century in Spanish America.”. In: Revista de estudios hispánicos 28 (2): 1994, 283-292.

Barreneche, Osvaldo. Crime and the Administration of Criminal Justice in Buenos Aires, Argentina, 1785-1853. Disertación de Doctorado, University of Arizona, 1997.

Borges, Jorge Luis. Obras completas. Edición crítica comentada por Rolando Costa Picazo e Irma Zangara. 3 vols. Buenos Aires: Emecé, 2009.

Borges, Jorge Luis y Adolfo Bioy Casares. Poesía gauchesca. México: Fondo de Cultura Económica, 1955.

Bourdieu, Pierre. "The Force of Law: Toward a Sociology of the Juridical Field." In: Hastings Law Journal 38: 1987, 805-853. 
Butler, Judith. "Replantear el universal: la hegemonía y los límites del formalismo". In: Contingencia, hegemonía, universalidad: diálogos contemporáneos en la izquierda. Buenos Aires: Fondo de Cultura Económica de Argentina, 2011, $19-50$.

Caimari, Lila. Apenas un delincuente: crimen, castigo y cultura en la Argentina, 18801955. Buenos Aires: Siglo Veintiuno Editores Argentina, 2004.

Chiaramonte, Jose Carlos. "La constitución antes de la constitución". In: Revista N, Buenos Aires, 14 Oct. 2013. http://www.clarin.com/ideas/constitucion-1853_0_ SJycxBQiDQg.html

Cover, Robert M. "The Supreme Court, 1982 Term -- Foreword: Nomos and Narrative”. In: Faculty Scholarship Series. Paper 2705. 1983.

Dabove, Juan Pablo. Nightmares of the Lettered City: Banditry and Literature in Latin America, 1816-1929. Pittsburgh, PA: University of Pittsburgh Press, 2007.

Dimock, Wai-Chee. Residues of Justice: Literature, Law, Philosophy. Berkeley: University of California Press, 1996.

Friedman. Lawrence. "Law, Lawyers, and Popular Culture". In: The Yale Law Journal 98 (8): 1989. 1579-1606. http://links.jstor.org/sici?sici=00440094\% 28198906\%2998\%3A8\%3C1579\%3ALLAPC\%3E2.0.CO\%3B2-1

Friedman. Lawrence. "Legal Culture and Social Development". In: Law \& Society Review 4 (1): 1969, 29-44. http://www.jstor.org/stable/3052760

Gamerro, Carlos. "El nacimiento de la literatura argentina". In: El nacimiento de la literatura argentina y otros ensayos. Buenos Aires: Grupo Editorial Norma.

Haritatos, P. "Invisible Strengths: the Greek Experience". In: Globalization: Critical Perspectives, Editado por Gernot Kohler y Emilio Jose Chaves, n.p., New York: Nova Science, 2003.

Hernández, José. Martín Fierro. Argentina: Ediciones Ayer, 1999. 
Kahn, Paul. The Cultural Study of Law: Reconstructing Legal Scholarship. Chicago: University of Chicago Press, 1999.

Kohan, Martín. "El desertor de fierro”. In: Diario Perfil, 10 Agosto 2013.

Ludmer, Josefina. "Los escándalos de Moreira".In: Las culturas de fin de siglo en América Latina, Rosario: Beatriz Viterbo, 1994, 102-112.

Ludmer, Josefina. El género gauchesco. Buenos Aires: Eterna Cadencia, 2012.

Martínez Estrada, Ezequiel. Muerte y transfiguración de Martín Fierro; ensayo deinterpretación de la vida argentina. México: Fondo de Cultura Económica, 1958.

Piglia, Ricardo. “Teoría del complot”. In: Plácidos domingos. 4-15. http://70.32.114.117/gsdl/collect/revista/index/assoc/HASHae5e/6be0ea57. dir/r23_04nota.pdf

Rama, Ángel. La ciudad letrada. Santiago (Xile): Tajamar, 2004.

Rodríguez, Fermín. Un desierto para la nación. Buenos Aires: Eterna Cadencia, 2010.

Rodríguez Molas, Ricardo. Historia social del gaucho. Buenos Aires: Ediciones Marú, 1968.

Sarlo, Beatriz. La pasión y la excepción. Buenos Aires: Siglo Veintiuno Editores Argentina, 2003.

Sarlo, Beatriz y María Teresa Gramuglio. José Hernández. Buenos Aires: CEAL, 1968.

Silbey, Susan. "Legal Culture and Cultures of Legality". In: Sociology of Culture: A Handbook, editado por John R. Hall, Laura Grindstaff y Ming-cheng Lo, Routledge, 2010, 470-479. 
